\title{
A Modified HOSM Controller Applied to an ABS Laboratory Setup with Adaptive Parameter
}

\author{
Claudia Carolina Vaca García $\mathbb{D D}^{1}{ }^{1}$ Luis Adrián Ferré Covantes $\left(\mathbb{D},{ }^{1}\right.$ \\ Antonio Navarrete Guzmán $\mathbb{D}^{2,3}$ Claudia Verónica Vera Vaca $\mathbb{D}^{1},{ }^{4}$ \\ and Cuauhtémoc Acosta Lúa $\mathbb{D}^{1,5}$ \\ ${ }^{1}$ Departamento de Ciencias Tecnológicas, Universidad de Guadalajara, Centro Universitario de la Ciénega, \\ Av. Universidad 1115, Ocotlán 47820, Jalisco, Mexico \\ ${ }^{2}$ Department of Electrical and Electronic Engineering at the National Technological Institute of Mexico Campus Tepic, \\ Av. Tecnológico 2595, Tepic 63175, Nayarit, Mexico \\ ${ }^{3}$ Academic Unit of Basic Sciences and Engineering of the Autonomous University of Nayarit, City of Culture "Amado Nervo ", \\ Tepic, Nayarit, Mexico \\ ${ }^{4}$ Department of Industrial Engineering at the National Technological Institute of Mexico Campus Ocotlán, Av. Tecnológico S/N, \\ Ocotlán 47829, Jalisco, Mexico \\ ${ }^{5}$ Center of Excellence DEWS, University of L'Aquila, Via Vetoio, Loc. Coppito, L'Aquila 67100, Italy
}

Correspondence should be addressed to Cuauhtémoc Acosta Lúa; cuauhtemoc.acosta@cuci.udg.mx

Received 12 May 2021; Accepted 11 June 2021; Published 23 June 2021

Academic Editor: M Syed Ali

Copyright (c) 2021 Claudia Carolina Vaca García et al. This is an open access article distributed under the Creative Commons Attribution License, which permits unrestricted use, distribution, and reproduction in any medium, provided the original work is properly cited.

\begin{abstract}
The antilock braking system (ABS) is an electromechanical device whose controller is challenging to design because of its nonlinear dynamics and parameter uncertainties. In this paper, an adaptive controller is considered under the assumption that the friction coefficient is unknown. A modified high-order sliding-mode controller is designed to enhance the controller performance. The controller ensures tracking of the desired reference and identifies the unknown parameter, despite parametric variations acting on the real system. The stability proof is done using the Lyapunov approach. Some numerical and experimental tests evaluate the controller on a mechatronic system that represents a quarter-car model.
\end{abstract}

\section{Introduction}

The antilock braking system (ABS) in the actual vehicles is a mechatronic system that helps the driver to maintain control of the vehicle during emergency braking by preventing the wheels from lock-up. The ABS is designed to increase the braking efficiency and maintain the vehicle's maneuverability, reducing the driving instability, obtaining maximum wheel grip on the surface while the vehicle is braking, and decreasing the braking distance.

During the last decade, ABSs were improved considering more advanced technologies and more sophisticated control strategies. However, it is essential to highlight that the tireroad friction coefficient is one of the most critical parameters since friction is the mechanism for transmitting external forces to the vehicle. These friction forces are the primary forces affecting the planar vehicle motion. From a physical point of view, these forces are limited by the road surface coefficient of friction $\mu$ and the instantaneous tire normal forces. The condition (i.e., the value of $\mu$ ) of the road surface, even if regular, could negatively influence the vehicle motion since the road could be dangerously slippery (e.g., due to water or ice). In practical cases, the road condition is one of the most relevant parameters causing the driving control loss. In particular, the knowledge of the real tire-road friction coefficient is critical to apply active control actions properly. Therefore, its precise estimation increases the efficiency of the control system considerably. There is 
literature regarding the modeling [1] and the estimation [2] of the tire-road friction coefficient. These studies deal mainly with identifying the tire-road friction coefficient to improve the vehicle acceleration or deceleration [3-13]. However, today, the $A B S$ is very important for passenger car safety, so further efforts should be made to continue studying it. In fact, novel techniques can be applied such as those in [14-16].

In this article, the ABS laboratory setup, manufactured by Inteco Ltd., has been used to test the proposed controller. This setup represents a quarter-car model [17], and it consists of two rolling wheels. Earlier works about nonlinear controllers were considered. These works are mainly based on the assumption that the information of all sensors is available for measurement. In [18], an experimental comparison between PID and nonlinear stabilizing controllers is presented, and in [19], an event-triggered control is proposed. Also, the sliding mode control strategies are analyzed in $[20,21]$. Other works deal with intelligent control techniques such as adaptive neuro-fuzzy $[22,23]$, neurofuzzy techniques [24], or other fuzzy controllers [25, 26].

In this paper, an adaptive controller using the modified HOSM is designed for the ABS laboratory setup. The controllers ensure tracking of the desired reference, even in uncertainties in the friction coefficient and external perturbations. At the same time, the identification of the friction coefficient parameter is developed. The stability proof using the appropriate function of Lyapunov and the performance of the controller is evaluated by some numerical simulations and experimental tests on the ABS laboratory setup.

The paper is organized as follows. Section 2 introduces the description and the mathematical model of the experimental ABS laboratory setup. In Section 3, the main contributions are presented. Section 4 presents some numerical simulation and real-time tests on the ABS laboratory setup. Some comments conclude the paper.

\section{Mathematical Model of the ABS Laboratory Setup}

The ABS laboratory setup describes the essential dynamics of a quarter-car model. It consists of two rolling wheels: the lower aluminum wheel emulates the road motion, and the upper plastic wheel simulates the vehicle wheel. In order to accelerate the lower wheel, a DC motor is coupled on it, whereas the upper wheel is equipped with a disk-brake system. Encoders on the wheels allow determining the positions and velocities of the two wheels, using numerical differentiation. This laboratory setup, manufactured by Inteco Ltd., and shown in Figure 1, preserves the fundamental characteristics of an actual ABS system in the range of $0-70 \mathrm{~km} / \mathrm{h}[17]$.

The dynamic equations of the ABS laboratory setup are obtained from Figure 1 and are currently used in the literature [27-30]. The braking torque $T_{b}$ is used as a control variable, and it acts on the upper wheel. Additionally, the tangential braking force $F_{t}$ represents the tractive force generated at the contact between the upper and lower wheel.
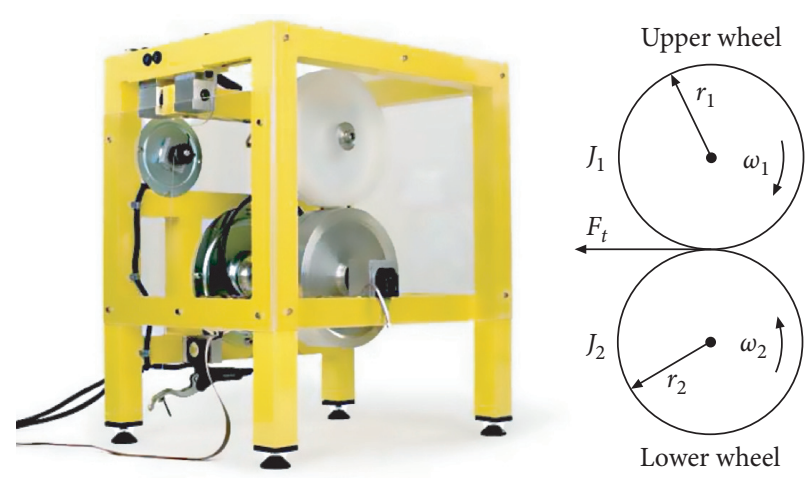

Figure 1: The ABS laboratory setup and its scheme.

$$
\begin{aligned}
& \dot{\omega}_{1}=\frac{r_{1}}{J_{1}} F_{t}-\frac{1}{J_{1}}\left(d_{1} \omega_{1}+T_{b}\right), \\
& \dot{\omega}_{2}=-\frac{r_{2}}{J_{2}} F_{t}-\frac{1}{J_{2}} d_{2} \omega_{2},
\end{aligned}
$$

where $\omega_{1}$ and $\omega_{2}$ are the angular velocities of the upper and lower wheels, respectively, whose inertia moments are $J_{1}$ and $J_{2}$ and whose radii are $r_{1}$ and $r_{2}$. Furthermore, $d_{1}$ and $d_{2}$ are the viscous friction coefficients of the upper and lower wheel.

The braking torque $T_{b}$ is modeled by a first-order equation [17]:

$$
\dot{T}_{b}=c\left(-T_{b}+b(u)\right)
$$

where $c>0$ is a constant, $u \in[0,1]$ is the control input, and $b(u)$ describes the relation between $u$ and the input applied to the DC motor. This relation can be approximated by an equation similar to the brake pedal model in an automobile $[27,31,32]$ :

$$
b(u)= \begin{cases}b_{1} u-b_{0}, & \text { if } u \geq u_{0}, \\ 0, & \text { if } u<u_{0},\end{cases}
$$

where $u_{0}$ is the threshold of the brake driving system.

On the other side, the tractive force $F_{t}$ is proportional, via the tire-road friction coefficient $\mu \in[0,1]$, to the normal load of the vehicle and is a nonlinear function of the longitudinal wheel slip.

$$
\lambda=\frac{r_{2} \omega_{2}-r_{1} \omega_{1}}{r_{2} \omega_{2}}=\frac{v_{x}-v_{w}}{v_{x}} .
$$

Under normal operation conditions, the wheel velocity $v_{w}$ matches the vehicle forward velocity $v_{x}$ and $\lambda=0$. When the braking is applied, $v_{w}$ tends to be lower than $v_{x}>0$ (remaining nonnegative), a slippage $\lambda>0$ occurs, and a tractive force $F_{t}$ is generated at the contact point, whose magnitude is given by

$$
F_{t}=\mu D \varphi(\lambda)=\theta \varphi(\lambda), \quad \theta=\mu D,
$$

where $\varphi(\lambda)$ represents the force $F_{t}$ normalized with respect to $\theta$ and $D$ is the force peak value:

$$
\varphi(\lambda)=\sin (C \arctan (B \lambda)),
$$


with $B$ being the stiffness factor and $C$ being the shape factor. The parameters $B, C$, and $D$ are determined to match the experimental data.

Remark 1. Various models are available in the literature to model the tire behavior, for example, the so-called Pacejka's "magic formula" [33] which approximates the response curve of the braking process based on experimental data. It is widely used and allows working with a wide range of values, including the linear and nonlinear regions of the tire characteristics.

Hence, considering (5), the dynamic equation of the ABS laboratory setup (1) can be rewritten as

$$
\begin{aligned}
& \dot{\omega}_{1}=\frac{r_{1}}{J_{1}} \theta \varphi(\lambda)-\frac{1}{J_{1}}\left(d_{1} \omega_{1}+T_{b}\right), \\
& \dot{\omega}_{2}=-\frac{r_{2}}{J_{2}} \theta \varphi(\lambda)-\frac{1}{J_{2}} d_{2} \omega_{2} .
\end{aligned}
$$

To design the controller, it is assumed that $v_{x}>0$. The output to be controlled is the wheel slip $\lambda$, and the control aim is to design a controller such that $\lambda$ tracks in finite time a constant reference $\lambda_{\text {ref }}$ in the presence of parameter uncertainties inherent to the ABS laboratory setup.

\section{Design of an Adaptive Controller for the ABS Laboratory Setup}

In this section, a modified high-order sliding-mode (MHOSM) controller is designed to force the error,

$$
e_{\lambda}=\lambda-\lambda_{\text {ref }}
$$

to zero in finite time, even in the presence of variations of $\theta$. The control law needs a control reference $\lambda_{\text {ref }}$. Hence, instead of considering the wheel slip as the control variable, an auxiliary slip velocity $v_{s}=v_{x}-v_{\omega}=\lambda v_{x}$ will be used $[29,30,32]$. Then, the slip velocity reference is given by $v_{s, \text { ref }}=\lambda_{\text {ref }} v_{x}$. Therefore, the slip velocity error is defined as

$$
\begin{aligned}
& e_{v}=v_{s}-v_{s, \text { ref }}=\left(1-\lambda_{\text {ref }}\right) v_{x}-v_{\omega}, \\
& e_{v}=\left(1-\lambda_{\text {ref }}\right) r_{2} \omega_{2}-r_{1} \omega_{1},
\end{aligned}
$$

and the dynamics

$$
\begin{aligned}
\dot{e}_{v} & =\left(1-\lambda_{\text {ref }}\right) \dot{v}_{x}-\dot{v}_{\omega}=\left(1-\lambda_{\text {ref }}\right) r_{2} \dot{\omega}_{2}-r_{1} \dot{\omega}_{1} \\
& =-k\left(\lambda_{\text {ref }}\right) \theta \varphi(\lambda)+\frac{r_{1}}{J_{1}} d_{1} \omega_{1}-\left(1-\lambda_{\text {ref }}\right) \frac{r_{2}}{J_{2}} d_{2} \omega_{2}+\frac{r_{1}}{J_{1}} T_{b},
\end{aligned}
$$

with $k\left(\lambda_{\text {ref }}\right)=\left(r_{1}^{2} / J_{1}\right)+\left(1-\lambda_{\text {ref }}\right)\left(r_{2}^{2} / J_{2}\right)$.

However, the friction coefficient $\mu$ is a parameter that, in real cases, may vary considerably, according to the road and tire conditions. Also, the parameter $D$ (value of force peak of Pacejka's magic formula) depends on the tire condition. In this article, a controller in which the parameter $\theta$ is constant and unknown is proposed. The next result solves the control problem in the case of uncertainty of this parameter.

Theorem 1. Consider the following assumption:

(i) The slip reference $\lambda_{\text {ref }}$ is a constant

(ii) The angular velocities $\omega_{1}, \omega_{2}$ are measurable

(iii) The parameter $\theta$ is constant and unknown

Then, the modified high-order sliding mode controller is proposed:

$$
\begin{aligned}
\dot{\hat{\omega}}_{1}= & -\frac{r_{1}}{J_{1}} \hat{\theta} \varphi(\lambda)-\frac{1}{J_{1}}\left(d_{1} \omega_{1}+T_{b}\right), \\
T_{b}= & \frac{J_{1}}{r_{1}} \hat{\chi}, \\
\hat{\chi}= & k\left(\lambda_{\text {ref }}\right) \hat{\theta} \varphi(\lambda)-\frac{r_{1}}{J_{1}} d_{1} \omega_{1}+\left(1-\lambda_{\text {ref }}\right) \frac{r_{2}}{J_{2}} d_{2} \omega_{2}-\alpha_{11}\left\lfloor e_{v}\right\rceil^{(1 / 2)} \\
& -\alpha_{12} e_{v}+\hat{x}_{v}, \\
\dot{\hat{x}}_{v}= & -\alpha_{2,1}\left\lfloor e_{v}\right\rceil^{0}-\alpha_{2,2} e_{v},
\end{aligned}
$$

with $\quad k\left(\lambda_{\text {ref }}\right)=\left(r_{1}^{2} / J_{1}\right)+\left(1-\lambda_{\text {ref }}\right)\left(r_{2}^{2} / J_{2}\right)$ and $\alpha_{11}, \alpha_{12}, \alpha_{21}, \alpha_{22}>0$ ensures that the tracking error (9) converges to zero in finite time [34] and the estimation error $\widetilde{\theta}=$ $\theta-\widehat{\theta}$ globally exponentially tends to zero along their derivatives.

Proof. Substituting the control input (11) into the dynamics of the slip velocity error (10), one obtains

$$
\begin{aligned}
\dot{e}_{v}= & -k\left(\lambda_{\text {ref }}\right) \theta \varphi(\lambda)+\frac{r_{1}}{J_{1}}\left(d_{1} \omega_{1}\right) \\
& -\left(1-\lambda_{\text {ref }}\right) \frac{r_{2}}{J_{2}}\left(d_{2} \omega_{2}\right)+\frac{r_{1}}{J_{1}}\left(\frac{J_{1}}{r_{1}} \widehat{\chi}\right) \\
= & \alpha_{11}\left\lfloor e_{v}\right\rceil^{(1 / 2)}-\alpha_{12} e_{v}+\widehat{x}_{v}-k\left(\lambda_{\text {ref }}\right) \varphi(\lambda) \widetilde{\theta} \\
\left(\begin{array}{c}
\dot{e}_{v} \\
\dot{\hat{x}}_{v}
\end{array}\right)= & \left(\begin{array}{c}
-\alpha_{11}\left\lfloor e_{v}\right\rceil^{(1 / 2)}-\alpha_{12} e_{v}+\widehat{x}_{v} \\
-\alpha_{21}\left\lfloor e_{v}\right\rceil^{0}-\alpha_{22} e_{v}
\end{array}\right)+\left(\begin{array}{c}
1 \\
0
\end{array}\right) \vartheta(\lambda) \widetilde{\theta}
\end{aligned}
$$

with $\vartheta(\lambda)=-k\left(\lambda_{\text {ref }}\right) \varphi(\lambda)$.

Let us consider the following Lyapunov function:

$$
\mathscr{V}=\mathscr{V}_{\xi}+\mathscr{V}_{\theta},
$$

with

$$
\begin{aligned}
& \mathscr{V}_{\xi}=\frac{1}{2} \xi^{T} P \xi \\
& \mathscr{V}_{\theta}=\frac{1}{2 \gamma} \tilde{\theta}^{2}
\end{aligned}
$$

with $\widetilde{\theta}=\theta-\widehat{\theta}$ and 


$$
\begin{aligned}
\xi & =\left(\begin{array}{c}
\left\lfloor e_{v}\right\rceil^{(1 / 2)} \\
e_{v} \\
\widehat{x}_{v}
\end{array}\right), \\
P & =\left(\begin{array}{ccc}
4 \alpha_{21}+\alpha_{11}^{2} & \alpha_{11} \alpha_{12} & -\alpha_{11} \\
\alpha_{11} \alpha_{12} & 2 \alpha_{22}+\alpha_{12}^{2} & -\alpha_{12} \\
-\alpha_{11} & -\alpha_{12} & 2
\end{array}\right) .
\end{aligned}
$$

Deriving (15) along the trajectories of the system,

$$
\begin{aligned}
& \dot{\mathscr{V}}_{\xi}=\xi^{T} P \dot{\xi}, \\
& \dot{\mathscr{V}}_{\theta}=\frac{1}{\gamma} \tilde{\theta} \dot{\tilde{\theta}},
\end{aligned}
$$

with

$$
\begin{aligned}
\dot{\xi}= & \left(\begin{array}{c}
\left\lfloor\left.\dot{e}_{v}\right|^{(1 / 2)}\right. \\
\dot{e}_{v} \\
\dot{\hat{x}}_{v}
\end{array}\right)=-\frac{1}{2\left|e_{v}\right|^{(1 / 2)} \Lambda_{1} \xi-\frac{1}{2} \Lambda_{2} \xi} \\
& +\frac{1}{2\left|e_{v}\right|^{(1 / 2)}} \Theta_{1} \widetilde{\theta}+\frac{1}{2} \Theta_{2} \widetilde{\theta}, \\
\Lambda_{1}= & \left(\begin{array}{ccc}
\alpha_{11} & 0 & -1 \\
0 & 0 & 0 \\
2 \alpha_{21} & 0 & 0
\end{array}\right), \\
\Lambda_{2}= & \left(\begin{array}{ccc}
\alpha_{12} & 0 & 0 \\
2 \alpha_{11} & 2 \alpha_{12} & -2 \\
0 & 2 \alpha_{22} & 0
\end{array}\right), \\
\Theta_{1}= & \left(\begin{array}{c}
\vartheta(\lambda) \\
0 \\
0
\end{array}\right), \\
\Theta_{2}= & \left(\begin{array}{c}
0 \\
2 \vartheta(\lambda) \\
0
\end{array}\right) .
\end{aligned}
$$

The derivative of $\mathscr{V}_{\xi}(15)$ is with $\dot{\mathscr{V}}_{\xi}=-\frac{1}{2\left|e_{v}\right|^{(1 / 2)}} \xi^{T} P \Lambda_{1} \xi-\frac{1}{2} \xi^{T} P \Lambda_{2} \xi+\frac{1}{2\left|e_{v}\right|^{(1 / 2)}} \xi^{T} P \Theta_{1} \tilde{\theta}+\frac{1}{2} \xi^{T} P \Theta_{2} \tilde{\theta}$,

$$
\begin{aligned}
P \Lambda_{1} & =\left(\begin{array}{ccc}
\alpha_{11}\left(\alpha_{11}^{2}+2 \alpha_{21}\right) & 0 & -\alpha_{11}^{2} \\
\alpha_{12}\left(\alpha_{11}^{2}-2 \alpha_{21}\right) & 0 & -\alpha_{11} \alpha_{12} \\
-\alpha_{11}^{2} & 0 & \alpha_{11}
\end{array}\right), \\
P \Lambda_{2} & =\left(\begin{array}{ccc}
\alpha_{12}\left(4 \alpha_{21}+3 \alpha_{11}^{2}\right. & 2 \alpha_{11}\left(\alpha_{12}^{2}-\alpha_{22}\right) & -2 \alpha_{11} \alpha_{12} \\
\alpha_{11}\left(4 \alpha_{22}+3 \alpha_{12}^{2}\right) & 2 \alpha_{12}\left(\alpha_{12}^{2}+\alpha_{22}\right) & -2\left(2 \alpha_{22}+\alpha_{12}^{2}\right) \\
-3 \alpha_{11} \alpha_{12} & 4 \alpha_{22}-2 \alpha_{12}^{2} & 2 \alpha_{12}
\end{array}\right), \\
P \Theta_{1}= & \left(\begin{array}{c}
4 \alpha_{21}+\alpha_{11}^{2} \\
\alpha_{11} \alpha_{12} \\
-\alpha_{11} \\
\alpha_{11} \alpha_{12} \\
2 \alpha_{22}+\alpha_{12}^{2} \\
-\alpha_{12}
\end{array}\right) \vartheta(\lambda),
\end{aligned}
$$


Analyzing the first term of (19), i.e., $-\left(1 / 2\left|e_{v}\right|^{(1 / 2)}\right) \xi^{T} P \Lambda_{1} \xi$ can be rewritten as

$$
\begin{aligned}
-\frac{1}{2\left|e_{v}\right|^{(1 / 2)}} \xi^{T} P \Lambda_{1} \xi= & \left(\begin{array}{c}
\left\lfloor e_{v}\right\rceil^{(1 / 2)} \\
e_{v} \\
\hat{x}_{v}
\end{array}\right)^{T}\left(\begin{array}{ccc}
\alpha_{11}\left(\alpha_{11}^{2}+2 \alpha_{21}\right) & 0 & -\alpha_{11}^{2} \\
\alpha_{12}\left(\alpha_{11}^{2}-2 \alpha_{21}\right) & 0 & -\alpha_{11} \alpha_{12} \\
-\alpha_{11}^{2} & 0 & \alpha_{11}
\end{array}\right)\left(\begin{array}{c}
\left\lfloor e_{v}\right\rceil^{(1 / 2)} \\
e_{v} \\
\hat{x}_{v}
\end{array}\right) \\
= & -\frac{1}{2\left|e_{v}\right|^{(1 / 2)}}\left(\begin{array}{c}
\left\lfloor e_{v}\right\rceil^{(1 / 2)} \\
e_{v} \\
\hat{x}_{v}
\end{array}\right)^{T}\left(\begin{array}{ccc}
\alpha_{11}\left(\alpha_{11}^{2}+2 \alpha_{21}\right) & -\alpha_{11}^{2} \\
0 & 0 & -\frac{1}{2} \alpha_{11} \alpha_{12} \\
-\alpha_{11}^{2} & -\frac{1}{2} \alpha_{11} \alpha_{12} & \alpha_{11}
\end{array}\right)\left(\begin{array}{c}
\left\lfloor e_{v}\right\rceil^{(1 / 2)} \\
e_{v} \\
\hat{x}_{v}
\end{array}\right) \\
& -\frac{1}{2\left|e_{v}\right|^{(1 / 2)}} \alpha_{12}\left(\alpha_{11}^{2}-2 \alpha_{21}\right)\left\lfloor e_{v}\right\rceil^{(1 / 2)} e_{v} .
\end{aligned}
$$

If

$$
-\frac{1}{2\left|e_{v}\right|^{(1 / 2)}} \alpha_{12}\left(\alpha_{11}^{2}-2 \alpha_{21}\right)\left\lfloor e_{v}\right\rceil^{(1 / 2)} e_{v}=-\frac{1}{2} \alpha_{12}\left(\alpha_{11}^{2}-2 \alpha_{21}\right)\left(\left\lfloor e_{v}\right\rceil^{(1 / 2)}\right)^{2}
$$

the first term in matrix form is written as

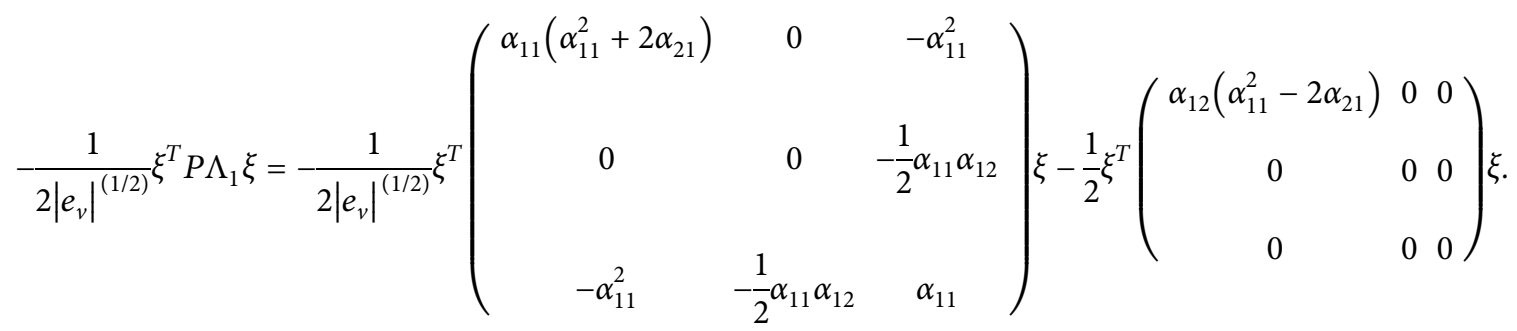

Similarly, the second term of (19) is $-(1 / 2) \xi^{T} P \Lambda_{2} \xi$ and can be evaluated as

$$
\begin{aligned}
& -\frac{1}{2} \xi^{T} P \Lambda_{2} \xi=-\frac{1}{2}\left(\begin{array}{c}
\left\lfloor e_{v}\right\rceil^{(1 / 2)} \\
e_{v} \\
\hat{x}_{v}
\end{array}\right)^{T}\left(\begin{array}{ccc}
\alpha_{12}\left(4 \alpha_{21}+3 \alpha_{11}^{2}\right) & 2 \alpha_{11}\left(\alpha_{12}^{2}-\alpha_{22}\right) & -2 \alpha_{11} \alpha_{12} \\
\alpha_{11}\left(4 \alpha_{22}+3 \alpha_{12}^{2}\right) & 2 \alpha_{12}\left(\alpha_{22}+\alpha_{12}^{2}\right) & -2\left(2 \alpha_{22}+\alpha_{12}^{2}\right) \\
-3 \alpha_{11} \alpha_{12} & 4 \alpha_{22}-2 \alpha_{12}^{2} & 2 \alpha_{12}
\end{array}\right)\left(\begin{array}{c}
\left\lfloor e_{v}\right\rceil^{(1 / 2)} \\
e_{v} \\
\hat{x}_{v}
\end{array}\right) \\
& =-\frac{1}{2} \xi^{T}\left(\begin{array}{ccc}
\alpha_{12}\left(4 \alpha_{21}+3 \alpha_{11}^{2}\right) & 0 & 0 \\
0 & 2 \alpha_{12}\left(\alpha_{22}+\alpha_{12}^{2}\right) & -2 \alpha_{12}^{2} \\
0 & -2 \alpha_{12}^{2} & 2 \alpha_{12}
\end{array}\right) \xi \\
& =-\frac{1}{2}\left[\alpha_{11}\left(5 \alpha_{12}^{2}+2 \alpha_{22}\right)\left\lfloor e_{v}\right\rceil^{(1 / 2)} e_{v}-5 \alpha_{11} \alpha_{12}\left\lfloor e_{v}\right\rceil^{(1 / 2)} \widehat{x}_{v}\right]
\end{aligned}
$$


Since $\left\lfloor e_{v}\right\rceil^{(1 / 2)} e_{v}=\left(1 /\left|e_{v}\right|^{(1 / 2)}\right) e_{v}^{2}$ and $\left\lfloor e_{v}\right\rceil^{(1 / 2)} \widehat{x}_{v}=(1 / \mid$ $\left.\left.e_{v}\right|^{(1 / 2)}\right) e_{v} \widehat{x}_{v}$, the second term is presented in the matrix form:

$$
\begin{gathered}
-\frac{1}{2} \xi^{T} P \Lambda_{2} \xi=-\frac{1}{2} \xi^{T}\left(\begin{array}{ccc}
\alpha_{12}\left(4 \alpha_{21}+3 \alpha_{11}^{2}\right) & 0 & 0 \\
0 & 2 \alpha_{12}\left(\alpha_{22}+\alpha_{12}^{2}\right) & -2 \alpha_{12}^{2} \\
0 & -2 \alpha_{12}^{2} & 2 \alpha_{12}
\end{array}\right) \xi \\
-\frac{1}{2\left|e_{v}\right|^{(1 / 2)} \xi^{T}}\left(\begin{array}{ccc}
0 & 0 & 0 \\
0 & \alpha_{11}\left(5 \alpha_{12}^{2}+2 \alpha_{22}\right) & -\frac{5}{2} \alpha_{11} \alpha_{12} \\
0 & -\frac{5}{2} \alpha_{11} \alpha_{12} & 0
\end{array}\right) \xi .
\end{gathered}
$$

Following with the terms of (19), one analyzes the term $\left(1 / 2\left|e_{v}\right|^{(1 / 2)}\right) \xi^{T} P \Theta_{1} \tilde{\theta}$ :

$\frac{1}{2\left|e_{v}\right|^{(1 / 2)}} \xi^{T} P \Theta_{1} \tilde{\theta}=\frac{1}{2\left|e_{v}\right|^{(1 / 2)}}\left(\begin{array}{c}\left\lfloor\left. e_{v}\right|^{(1 / 2)}\right. \\ e_{v} \\ \hat{x}_{v}\end{array}\right)^{T}\left(\begin{array}{c}\left(4 \alpha_{21}+\alpha_{11}^{2}\right) \\ \alpha_{11} \alpha_{12} \\ -\alpha_{11}\end{array}\right) \vartheta(\lambda) \tilde{\theta}$,

$$
\frac{1}{2} \xi^{T} P \Theta_{2} \widetilde{\theta}=\frac{1}{2}\left(\begin{array}{c}
\left\lfloor e_{v}\right\rceil^{(1 / 2)} \\
e_{v} \\
\hat{x}_{v}
\end{array}\right)^{T}\left(\begin{array}{c}
2 \alpha_{11} \alpha_{12} \\
2\left(2 \alpha_{22}+\alpha_{12}^{2}\right) \\
-2 \alpha_{12}
\end{array}\right) \vartheta(\lambda) \widetilde{\theta}
$$

Using (23), (25), (26), and (27), one rewrites (19) as

and the term $(1 / 2) \xi^{T} P \Theta_{2} \tilde{\theta}$ :

$$
\begin{aligned}
& \dot{\mathscr{V}}_{\xi}=-\frac{1}{2\left|e_{v}\right|^{(1 / 2)}} \xi^{T}\left(\begin{array}{ccc}
\alpha_{11}\left(\alpha_{11}^{2}+2 \alpha_{21}\right) & 0 & -\alpha_{11}^{2} \\
0 & \alpha_{11}\left(5 \alpha_{12}^{2}+2 \alpha_{22}\right) & -3 \alpha_{11} \alpha_{12} \\
-\alpha_{11}^{2} & -3 \alpha_{11} \alpha_{12} & \alpha_{11}
\end{array}\right) \xi \\
& -\frac{1}{2} \xi^{T}\left(\begin{array}{ccc}
\alpha_{12}\left(4 \alpha_{11}^{2}+2 \alpha_{21}\right) & 0 & 0 \\
0 & 2 \alpha_{12}\left(\alpha_{22}+\alpha_{12}^{2}\right) & -2 \alpha_{12}^{2} \\
0 & -2 \alpha_{12}^{2} & 2 \alpha_{12}
\end{array}\right) \xi \\
& +\frac{1}{2\left|e_{v}\right|^{(1 / 2)}} \xi^{T}\left(\begin{array}{c}
4 \alpha_{21}+\alpha_{11}^{2} \\
\alpha_{11} \alpha_{12} \\
-\alpha_{11}
\end{array}\right) \vartheta(\lambda) \widetilde{\theta}+\frac{1}{2} \xi^{T}\left(\begin{array}{c}
2 \alpha_{11} \alpha_{12} \\
2\left(2 \alpha_{22}+\alpha_{12}^{2}\right) \\
-2 \alpha_{12}
\end{array}\right) \vartheta(\lambda) \widetilde{\theta}
\end{aligned}
$$


Finally, using $\dot{\mathscr{V}}_{\xi}(28)$ and $\dot{\mathscr{V}}_{\theta}(17)$, the derivative of the Lyapunov function (14) is

$$
\begin{aligned}
\dot{\mathscr{V}}= & \dot{\mathscr{V}}_{\xi}+\dot{\mathscr{V}}_{\theta}=-\frac{1}{\left|e_{v}\right|^{(1 / 2)}} \xi^{T} Q_{1} \xi-\xi^{T} Q_{2} \xi+\frac{1}{\left|e_{v}\right|^{(1 / 2)}} \xi^{T} R_{1} \widetilde{\theta} \\
& +\xi^{T} R_{2} \tilde{\theta}+\frac{1}{\gamma} \tilde{\theta} \dot{\tilde{\theta}}
\end{aligned}
$$

with

$$
\begin{aligned}
& Q_{1}=\frac{\alpha_{11}}{2}\left(\begin{array}{ccc}
\alpha_{11}^{2}+2 \alpha_{21} & 0 & -\alpha_{11} \\
0 & 5 \alpha_{12}^{2}+2 \alpha_{22} & 3 \alpha_{12} \\
-\alpha_{11} & -3 \alpha_{12} & 1
\end{array}\right), \\
& Q_{2}=\alpha_{12}\left(\begin{array}{ccc}
2 \alpha_{11}^{2}+\alpha_{21} & 0 & 0 \\
0 & \alpha_{22}+\alpha_{12}^{2} & -\alpha_{12} \\
0 & -\alpha_{12} & 1
\end{array}\right), \\
& R_{1}=\frac{1}{2}\left(\begin{array}{c}
4 \alpha_{21}+\alpha_{11}^{2} \\
\alpha_{11} \alpha_{12} \\
-\alpha_{11} \\
\alpha_{11} \alpha_{12} \\
2 \alpha_{22}+\alpha_{12}^{2} \\
-\alpha_{12}
\end{array}\right) .
\end{aligned}
$$

Since $\dot{\tilde{\theta}}=\dot{\theta}-\dot{\hat{\theta}}$, recalling that $\theta$ is constant, one gets

$$
\dot{\hat{\theta}}=\gamma\left(\frac{1}{\left|e_{v}\right|^{(1 / 2)}} \xi^{T} R_{1} \vartheta(\lambda)+\xi^{T} R_{2} \vartheta(\lambda)+k_{\theta} \frac{J_{1}^{2}}{r_{1}^{2}}\left(\dot{\omega}_{1}-\dot{\hat{\omega}}_{1}\right)^{2}\right) .
$$

Finally, substituting the equation (30) into (29), one obtains

$$
\begin{aligned}
\dot{\mathscr{V}} & =-\frac{1}{\left|e_{v}\right|^{(1 / 2)}} \xi^{T} Q_{1} \xi-\xi^{T} Q_{2} \xi-k_{\theta} \varphi(\lambda)^{2} \widetilde{\theta}^{2} \\
& \leq-\frac{1}{\left|e_{v}\right|^{(1 / 2)}} \lambda_{\min }^{Q_{1}}\|\xi\|^{2}-\lambda_{\min }^{Q_{2}}\|\xi\|^{2}-k_{\theta} \varphi(\lambda)_{\max }^{2} \tilde{\theta}^{2} .
\end{aligned}
$$

Then, $\dot{\mathscr{V}}$ is negative definite. Therefore, $e_{v}$ and $\tilde{\theta}$ tend to zero. Since the adaptive controller (12) ensures that $v_{s}$ tends to $v_{s, \text { ref }}$ in asymptotic time, one concludes that $\lambda$ also tends to $\lambda_{\text {ref }}$ in asymptotic time.

\section{Simulation Results}

In this section, some numerical results and real-time experimental results are shown, using the ABS laboratory setup controlled by a PC. The objective is to show the performance of the controller (11).

4.1. Numerical Simulation. To develop the numerical simulations, the coefficients of the ABS laboratory setup are given in Table 1 and the controller implemented in numerical simulations (11) are given in Table 2 . Also, the tests are done considering $\omega_{1}(0)=\omega_{2}(0)=178(\mathrm{rad} / \mathrm{s})$ $(1700 \mathrm{rpm})$, as initial conditions for (7). These conditions simulate a vehicle that runs at a speed of $65 \mathrm{~km} / \mathrm{h}$, and suddenly, the brake system is activated, sending a control signal to the actuator to start the braking process. It is worth noticing that, in this setup, the nominal value of the friction coefficient between the two wheels, given by the constructor, is $\mu_{0}=1$. Nevertheless, this coefficient may vary in practice, remaining close to this value.

The numerical simulations are summarized in Figures 2-5, where it can be seen that the proposed controller (11) ensures the performance of the system. Figure 3 shows the wheel velocity $v_{w}$ and the vehicle longitudinal velocity $v_{x}$. The wheel slip $\lambda$ and the tracking error $e_{\lambda}=$ $\lambda-\lambda_{\text {ref }}$ are shown in Figure 4 . The applied input $T_{b}$ is shown in Figure 2. Finally, the estimation $\hat{\theta}$ given by (29) and used in the controller (11) is shown in Figure 5, where the real value is $\theta=22.98 \mathrm{~N}$.

4.1.1. Real-Time Simulation. In this section, some real-time experimental results are shown, using the ABS laboratory setup controlled by a PC. The interested reader can find in [17] the details about the system hardware and the implementation of the proposed controller. The objective is to show the performance of the controller (11). The coefficients of the ABS laboratory setup are given in Table 1, and the controller (11) implemented in real-time simulation is given in Table 3.

The real-time simulation is shown in Figures 6-9. The braking phase of the ABS laboratory setup starts at $5.7 \mathrm{~s}$ and finishes at $7 \mathrm{~s}$. It is important to highlight that, after this braking phase, corresponding to the maximum braking efficiency, the performance is no longer relevant. Figure 7 shows the wheel velocity $v_{w}$ and the vehicle longitudinal velocity $v_{x}$. It can be observed that the control input $T_{b}$ applied to the ABS setup system, shown in Figure 6, reduces the velocities gradually to zero in approximately $1.8 \mathrm{~s}$. Figure 8 shows the behavior of the wheel slip $\lambda$, the wheel slip desired $\lambda_{\backslash r}$, and the tracking error $e_{\lambda}=\lambda-\lambda_{\text {ref }}$. Finally, Figure 9 shows the identification of the unknown parameter $\hat{\theta}$ and the estimation error $e_{\theta}=\theta-\hat{\theta}$. Note that, in the real-time simulation, the braking process can be considered concluded after about $1.1 \mathrm{~s}$. The reader can compare these results with those of Section 4.1. It can be noticed that these experimental results differ from the simulation results due to the unmodeled dynamics, parameters variations, etc., affecting the real system. This is particularly evident after 6.5 s, i.e., when the braking process can be considered concluded. 
TABLE 1: Coefficients and system variables for the ABS laboratory setup.

\begin{tabular}{lrr}
\hline$r_{1}$ & Radius of the upper wheel & $0.0995 \mathrm{~m}$ \\
$r_{2}$ & Radius of the lower wheel & $0.0990 \mathrm{~m}$ \\
$J_{1}$ & Upper wheel inertia moment & $7.54 \times 10^{-3} \mathrm{Kg} \mathrm{m}^{2}$ \\
$J_{2}$ & Lower wheel inertia moment & $2.56 \times 10^{-3} \mathrm{Kg} \mathrm{m}^{2}$ \\
$\beta_{1}$ & Upper wheel viscous friction coefficient & $118.74 \times 10^{-6} \mathrm{Kgm}^{2} / \mathrm{s}$ \\
$\beta_{2}$ & Lower wheel viscous friction coefficient & $214.68 \times 10^{-6} \mathrm{Kgm}^{2} / \mathrm{s}$ \\
$\mu$ & Friction coefficient between wheels & 1 \\
$b_{1}$ & Constant & 15.24 \\
$b_{0}$ & Constant & 6.21 \\
$c$ & Constant & $20.37 \mathrm{~s}^{-1}$ \\
$u_{0}$ & Constant & 0.415 \\
$B$ & Stiffness factor & 26.76 \\
$C$ & Shape factor & 1.13 \\
$D$ & Peak value & 22.98 \\
\hline
\end{tabular}

TABLe 2: Coefficients and system variables of controller (11) used in numerical simulations.

\begin{tabular}{lcc}
\hline$\gamma_{11}$ & Gain of the controllers (11) & 50 \\
$\gamma_{12}$ & Gain of the controllers (11) & 15 \\
$\gamma_{21}$ & Gain of the controllers (11) & 50 \\
$\gamma_{22}$ & Gain of the controllers (11) & 35.0 \\
$\gamma$ & Adaptive gain (15) & 0.025 \\
$k_{\theta}$ & Adaptive gain (29) & 2500 \\
\hline
\end{tabular}



Figure 2: Input control $T_{b}$. 


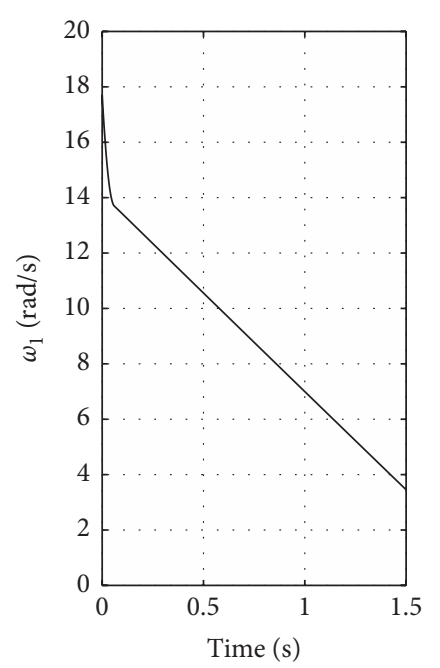

(a)



(b)

FIGURE 3: Angular velocity: (a) upper wheel $\omega_{1}$; (b) lower wheel $\omega_{2}$.

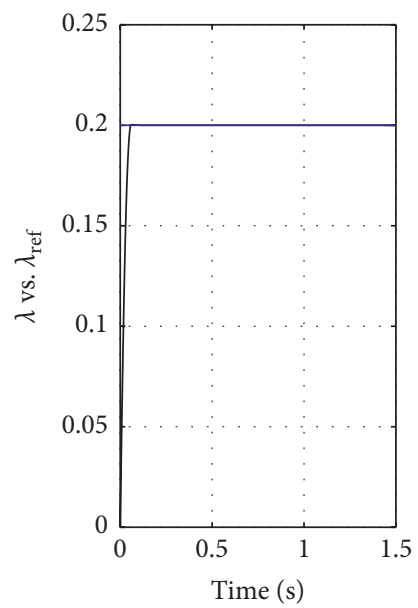

(a)

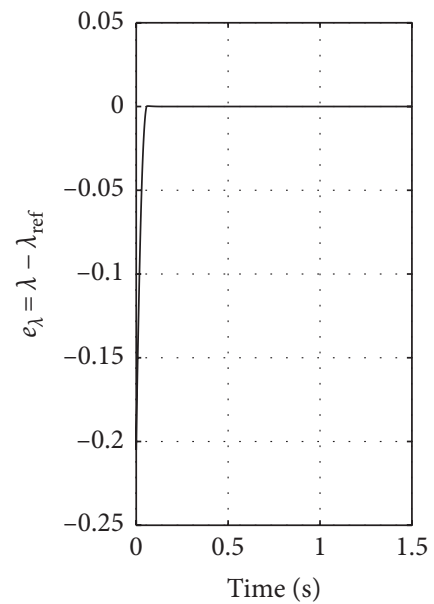

(b)

Figure 4: (a) Wheel slip $\lambda$ (black) and wheel slip reference $\lambda_{\text {ref }}$ (constant, blue). (b) Tracking error $e_{\lambda}=\lambda-\lambda_{\text {ref }}$.

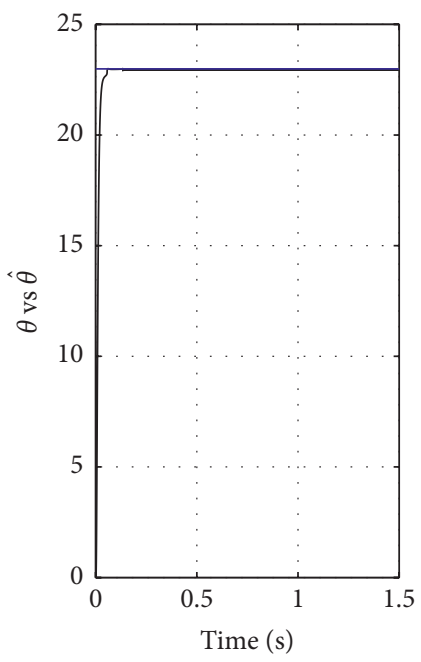

(a)

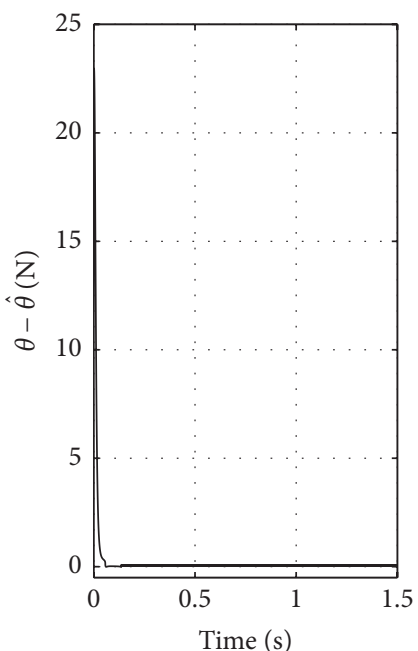

(b)

Figure 5: Friction coefficient between the wheels of the ABS laboratory setup: (a) real $\theta$ (blue) and estimated $\widehat{\theta}$ (black) and (b) estimation error $\theta-\widehat{\theta}$. 
TABLE 3: Coefficients and system variables of the controller (11) used in real application.

\begin{tabular}{llc}
\hline$\gamma_{11}$ & Gain of the controllers (11) & 15 \\
$\gamma_{12}$ & Gain of the controllers (11) & 12 \\
$\gamma_{21}$ & Gain of the controllers (11) & 1.7 \\
$\gamma_{22}$ & Gain of the controllers (11) & 0.5 \\
$\gamma$ & Adaptive gain (15) & 0.011 \\
$k_{\theta}$ & Adaptive gain (29) & 5000 \\
\hline
\end{tabular}

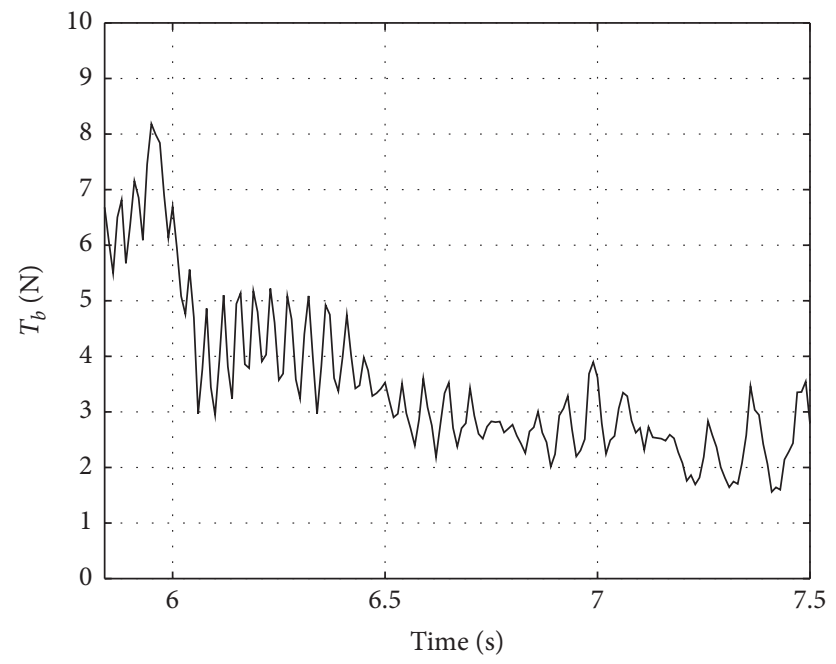

FIGURE 6: Input control $T_{b}$.

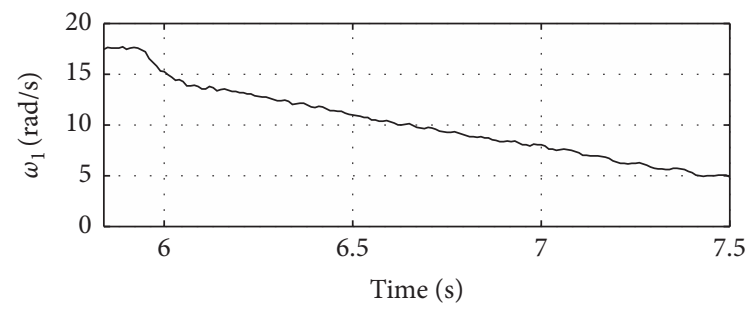

(a)

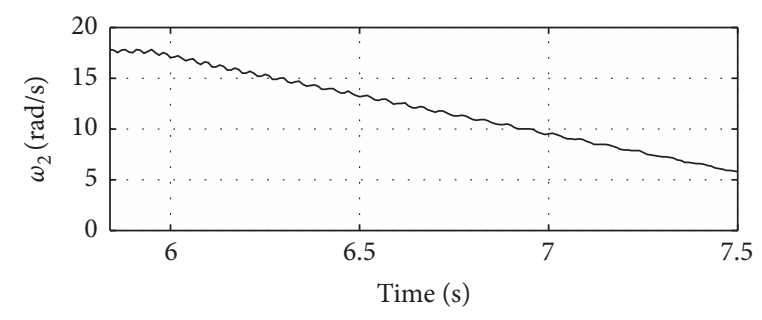

(b)

FIGURE 7: Angular velocity of the upper wheel $\omega_{1}$.



(a)

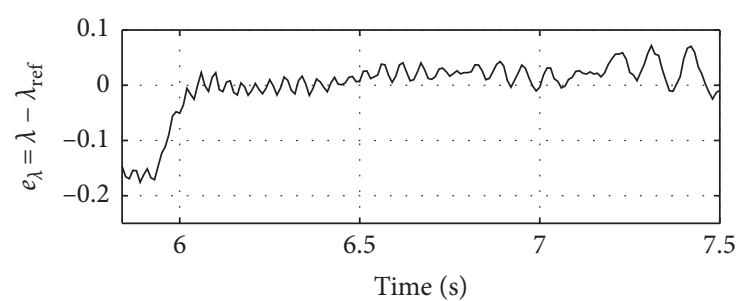

(b)

FIgURE 8: (a) Wheel slip $\lambda$ (black) and wheel slip reference $\lambda_{\text {ref }}$ (constant, blue). (b) Tracking error $e_{\lambda}=\lambda-\lambda_{\text {ref }}$. 


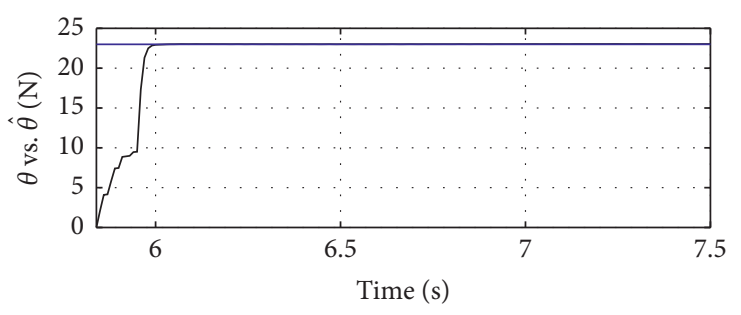

(a)

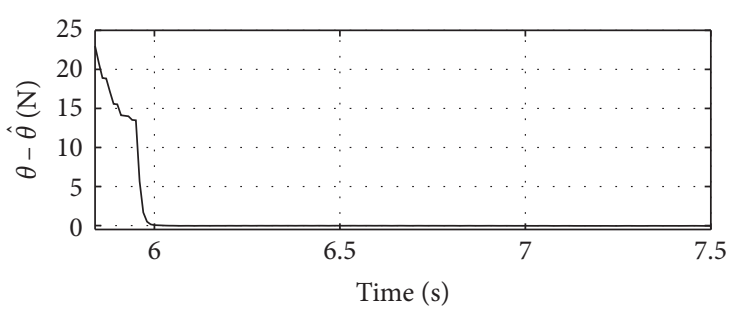

(b)

FIGURE 9: Friction coefficient between the wheels of ABS laboratory setup: (a) real $\theta$ (blue) and estimated $\hat{\theta}$ (black) and (b) estimation error $\theta-\widehat{\theta}$.

\section{Conclusions}

This paper presents a modified high-order sliding mode (HOSM) controller with parameter estimation applied to an ABS laboratory setup. The system emulates a quarter-car model. This controller provides estimations for the friction coefficient acting between the wheels. Once that parameter is estimated, the estimation can be used to determine the modified HOSM controller. This latter ensures tracking of the desired slip reference. The asymptotic stability is proven, and experimental tests show the effectiveness of the proposed controller. For the future, the work will be focused on finite-time sampled-data fuzzy control and the reliable fuzzy $H_{\infty}$ control of the ABS considering further dynamics, perturbations acting on the real system and parameter uncertainties.

\section{Data Availability}

The figures, tables, and other data used to support this study are included within the article.

\section{Conflicts of Interest}

The authors declare that there are no conflicts of interest regarding the publication of this paper.

\section{References}

[1] E. Velenis, P. Tsiotras, C. Canudas-de-Wit, and M. Sorine, "Dynamic tyre friction models for combined longitudinal and lateral vehicle motion," Vehicle System Dynamics, vol. 43, no. 1, pp. 3-29, 2005.

[2] F. Gustafsson, "Slip-based tire-road friction estimation," Automatica, vol. 33, no. 6, pp. 1087-1099, 1997.

[3] N. Patel, C. Edwards, and S. K. Spurgeon, "Comparative analysis of two non-linear observers for estimation of tyre/ road contact in the presence of imperfect measurements," IET Control Theory and Applications, vol. 4, no. 9, pp. 1501-1510, 2010.

[4] N. K. M'Sirdi, A. Rabhi, L. Fridman, J. Davila, and Y. Delanne, "Second order sliding-mode observer for estimation of vehicle dynamic parameters," International Journal Vehicle Design, vol. 48, no. 3/4, pp. 190-207, 2008.

[5] M. Amodeo, A. Ferrara, R. Terzaghi, and C. Vecchio, "Wheel slip control via second-order sliding-mode generation," IEEE Transactions on Intelligent Transportation Systems, vol. 11, no. 1, pp. 122-131, 2010.
[6] M. Doumiati, A. C. Victorino, A. Charara, and D. Lechner, "Onboard real-time estimation of vehicle lateral tire-road forces and sideslip angle," IEEE/ASME Transactions on Mechatronics, vol. 16, no. 4, pp. 601-614, 2011.

[7] J. J. Rath, K. C. Veluvolu, M. Defoort, and Y. C. Soh, "Higherorder sliding mode observer for estimation of tyre friction in ground vehicles," IET Control Theory and Applications, vol. 8, no. 6, pp. 399-408, 2014.

[8] J. J. Rath, K. C. Veluvolu, and M. Defoort, "Simultaneous estimation of road profile and tire road friction for automotive vehicle," IEEE Transactions on Vehicular Technology, vol. 64, no. 10, pp. 4461-4471, 2015.

[9] K. Han, E. Lee, M. Choi, and S. B. Choi, “Adaptive scheme for the real-time estimation of tire-road friction coefficient and vehicle velocity," IEEE/ASME Transactions on Mechatronics, vol. 22, no. 4, pp. 1508-1518, 2017.

[10] V. A. Jose and A. Francis, "Longitudinal tire force estimation using youla controller output observer," IEEE Control Systems Letters, vol. 2, no. 1, pp. 31-36, 2018.

[11] D. A. Aligia, G. A. Magallan, and C. H. De Angelo, "EV traction control based on nonlinear observers considering longitudinal and lateral tire forces," IEEE Transactions on Intelligent Transportation Systems, vol. 19, no. 8, pp. 25582571, 2018.

[12] W. Chen, D. Tan, L. Zhao, and Z. Wei, "Vehicle sideslip angle and road friction estimation using online gradient descent algorithm," IEEE Transactions on Vehicular Technology, vol. 67, no. 12, pp. 11475-11485, 2018.

[13] H. Guo, Z. Yin, D. Cao, H. Chen, and C. Lv, "A review of estimation for vehicle tire-road interactions toward automated driving," IEEE Transactions on Systems, Man, and Cybernetics: Systems, vol. 49, no. 1, pp. 14-30, 2019.

[14] R. Vadivel and Y. H. Joo, "Finite-time sampled-data fuzzy control for a non-linear system using passivity and passification approaches and its application," IET Control Theory and Applications, vol. 14, no. 8, pp. 1033-1045, 2020.

[15] R. Vadivel and Y. H. Joo, "Reliable fuzzy Ho control for permanent magnet synchronous motor against stochastic actuator faults," IEEE Transactions on Systems, Man, and Cybernetics: Systems, vol. 51, no. 4, pp. 2232-2245, 2021.

[16] R. Anbuvithya, S. D. Sri, R. Vadivel, N. Gunasekaran, and P. Hammachukiattikul, "Extended dissipativity and nonfragile synchronization for recurrent neural networks with multiple time-varying delays via sampled-data control," IEEE Access, vol. 9, pp. 31454-31466, 2021.

[17] Inteco User's Manual, The Laboratory Antilock Braking System Controlled from PC, Inteco Ltd., Crakow, Poland, 2006.

[18] M. Martinez-Gardea, I. J. Mares Guzman, S. Di Gennaro, and C. Acosta Lúa, "Experimental comparison of linear and nonlinear controllers applied to an Antilock braking system," 
in Proceedings of the IEEE Conference on Control Applications, pp. 71-76, Juan Les Antibes, France, October 2014.

[19] M. Martinez-Gardea, C. Acosta-Lúa, I. Vázquez-Álvarez, and S. Di Gennaro, "Event-triggered linear control design for an Antilock braking system," in Proceedings of the IEEE International Autumn Meeting on Power, Electronics and Computing (ROPEC 2015), pp. 1-6, Ixtapa, Mexico, November 2015.

[20] Y. Oniz, E. Kayacan, and O. Kaynak, "A dynamic method to forecast the wheel slip for antilock braking system and its experimental evaluation," IEEE Transactions on System, Man and Cybernetics-Part B: Cybernetics, vol. 39, no. 2, pp. 551560, 2009.

[21] R. E. Precup, S. V. Spătaru, M. B. Rădac et al., "Experimental results of model-based fuzzy control solutions for a laboratory Antilock braking system," Advances in Intelligent and Soft Computing, pp. 223-234, 2012.

[22] A. V. Topalov, E. Kayacan, Y. Oniz, and O. Kaynak, "Adaptive neuro-fuzzy control with sliding mode learning algorithm: application to Antilock braking system," in Proceedings of the 7th Asian Control Conference, pp. 1-6, Hong Kong, China, August 2009.

[23] A. V. Topalov, E. Kayacan, Y. Oniz, and O. Kaynak, "Neuro-fuzzy control of Antilock braking system using variable structure-systems based learning algorithm," in Proceedings of the International Conference on Adaptive and Intelligent Systems, pp. 1-6, Klagenfurt, AS, USA, September 2009.

[24] A. V. Topalov, Y. Oniz, E. Kayacan, and O. Kaynak, "Neuro-fuzzy control of antilock braking system using sliding mode incremental learning algorithm," Neurocomputing, vol. 74, no. 11, pp. 1883-1893, 2011.

[25] R. Precup, S. Preitl, M. Radac, E. M. Petriu, C. Dragos, and J. K. Tar, "Experimental-based teaching in advanced control engineering," IEEE Transaction on Education, vol. 61, no. 6, pp. 345-355, 2011.

[26] M. A. Khanesar, E. Kayacan, M. Teshnehlab, and O. Kaynak, "Extended kalman filter based learning algorithm for type-2 fuzzy logic systems and its experimental evaluation," IEEE Transactions on Industrial Electronics, vol. 59, no. 11, pp. 4443-4455, 2012.

[27] M. H. Al-Mola, M. Mailah, P. M. Samin, A. H. Muhaimin, and M. Y. Abdullah, "Performance comparison between sliding mode control and active force control for a nonlinear anti-lock brake system," WSEAS Transactions on Systems and Control, pp. 101-107, 2014.

[28] C. Acosta-Lúa, S. Di Gennaro, and M. E. Sánchez-Morales, "An adaptive controller applied to an anti-lock braking system laboratory," Dyna, vol. 83, no. 199, pp. 69-77, 2016.

[29] C. Acosta Lúa, S. Di Gennaro, and M. E. Sánchez Morales, "Nonlinear adaptive controller applied to an antilock braking system with parameters variations," International Journal of Control, Automation and Systems, vol. 15, no. 5, pp. 20432052, 2017.

[30] C. Acosta-Lúa, L.-A. Ferré-Covantes, C.-C. Vaca-García, and S. Di Gennaro, "Equivalent control and reaching law applied to ABS," in Proceedings of the IEEE International Autumn Meeting on Power, Electronics and Computing (ROPEC), pp. 1-6, Ixtapa, Mexico, November 2020.

[31] R. Bosch GmbH, Bosch Automotive Handbook, Bentley Publisher, 8th edition, 2011.

[32] C. Acosta Lúa, B. Castillo-Toledo, S. Di Gennaro, and M. Martinez Gardea, "Dynamic control applied to a laboratory antilock braking system," Mathematical Problems in Engineering, vol. 2015, Article ID 896859, 10 pages, 2015.
[33] H. Pacejka, Tyre and Vehicle Dynamics, Elsevier, Amsterdam, The Netherlands, 2006.

[34] J. A. Moreno and M. Osorio, "A Lyapunov approach to second-order sliding mode controllers and observers," in Proceedings of the 47th IEEE Conference on Decision and Control, pp. 2856-2861, Cancún, Mexico, December 2008. 